\title{
Drinking Water Quality in Malaysia: A Review on Its Current Status
}

\author{
Zurahanim Fasha Anual ${ }^{1 *}$, Wan Nurul Farah Wan Azmi ${ }^{1}$, Nurul Izzah Ahmad ${ }^{1}$, Noraishah Mohammad Sham ${ }^{1}$, \\ Wan Rozita Wan Mahiyuddin ${ }^{1}$, Yuvaneswary Veloo ${ }^{1}$ and Nor Aini Abdullah ${ }^{2}$ \\ ${ }^{1}$ Environmental Health Research Centre, Institute for Medical Research, National Institutes of Health, Malaysia \\ ${ }^{2}$ Infectious Disease Research Centre, Institute for Medical Research, National Institutes of Health, Malaysia
}

Submission: February 11, 2020; Published: April 09, 2020

*Corresponding author: Zurahanim Fasha Anual, Environmental Health Research Centre, Institute for Medical Research, National Institutes of Health, Malaysia

\section{Abstract}

Access to safe drinking water is fundamental to health. This review aims to assess the status of drinking water quality in Malaysia. Only peer-reviewed cross-sectional articles from January 2000 until May 2018 focusing on treated water and groundwater in Malaysia were included in this review. Based on the inclusion criteria, 30 articles were selected which measures water quality parameters in drinking water. Overall pooled mean for physical parameters ( $\mathrm{pH}$, turbidity, total suspended solids, total dissolved solids, temperature and conductivity) were within the reference levels established by the Malaysian National Drinking Water Quality Standard. In this review, the generally low number of available studies which fulfil the inclusion criteria means that interpretation of results must be exercised with caution. This review may provide policy makers with reliable scientific evidences for better management practice and it is hoped that the findings will support the planning of future researches in this field.

Keywords: Chemical contaminants; Microbiological contamination; Tap water; Water pollutants; Treated water

\section{Introduction}

According to the World Health Organization (WHO), access to safe water supply is basic human rights [1]. Since 1990, 2.6 billion people have gained access to improved drinking water of which $96 \%$ are urban population as opposed to $84 \%$ rural population (WHO and UNICEF 2015). Estimation by the [2] shows that 663 million people worldwide still use unimproved drinking water sources, including unprotected wells, springs and surface water. Almost half of them live in sub-Saharan Africa whereas one fifth live in Southern Asia.

Malaysia is a tropical country blessed with abundant rainfall throughout the year, yet the nation is still experiencing water shortage and issues with water quality. The Department of Environment in the 2017 Annual Report stated that almost $63.94 \%$ (144) out of 477 rivers in Malaysia are categorized as Class II whereas $30.19 \%$ (144) are in Class III. Major pollutants in the rivers are biochemical oxygen demand (BOD) from sewage, agrobased and manufacturing industries, ammoniacal nitrogen from animal farming and domestic sewage as well as suspended solids from earthworks and land-clearing activities [3]. In Malaysia, water supply for domestic use derives $99 \%$ from surface water such as rivers whereas the remaining $1 \%$ originates from groundwater [4]. The most common source of drinking water in Malaysia is tap water, bottled drinking water as well as bottled mineral water [5]. Surface water extracted from Sungai Langat, Sungai Selangor and Sungai Kinta in West Coast Peninsular Malaysia are amongst water supply most generally utilized for drinking water [6]. On the other hand, states like Kelantan, Terengganu, Pahang, Perlis, Kedah, Sabah and Sarawak use groundwater as drinking water source [7].

In Malaysia, extensive studies have been conducted on drinking water quality but to our knowledge, no extensive review on status of drinking water quality have been done thus far. While some studies focused on microbiological, chemical, elemental or physical parameters either in treated water, filtered water, ground water or mineral water, however, it is difficult to come to a conclusion with regards to the status of overall drinking water quality in this country.

Hence, this review is undertaken to systematically review on drinking water quality studies conducted in Malaysia and compile all available data on its status in this country, to compare with the National Drinking Water Quality Standards (NDWQS) and finally to provide the policy maker with scientific evidence for better management practice. 


\section{Materials and Methods}

All peer reviewed cross-sectional studies which were assessing water quality in Malaysia based on the parameters in the National Drinking Water Quality Guidelines (NDWQS) were sought for. As our stakeholders were interested in the current status of Malaysian water quality, the search was limited to the more recent studies from year 2000 to May 2018. Studies were eligible for inclusion if the water samples included treated water (filtered, bottled, tap water) and groundwater (bottled mineral water, well water) that were used for drinking water. Only fieldbased studies of observational longitudinal or cross-sectional design that use primary or secondary data and risk assessment involving water quality parameters (whether or not included in the NDWQS) conducted in Malaysia were considered. At least one water quality parameter analysed in the studies should be included in NDWQS. The drinking water quality parameters consisted of various groups, which were physical parameters, microbiological, elementals and other chemicals.

The exclusion criteria were narrative or systematic reviews, conference abstracts, proceedings, reports, theses and keynote texts. Surface water and ground water (not used for drinking purposes) were excluded for this review. Apart from literature which were published in English and Malay language, the literature which were published in other languages such as Chinese and Indian languages were also included. We sought help from Chinese and Indian colleagues to interpret the abstract and the content of the potential eligible report.

\section{Search strategy and quality assessment}

Table 1: Search strategy for identification of studies.

\begin{tabular}{|c|}
\hline Keywords \\
\hline $\begin{array}{c}\text { 1. 'tap water' OR 'raw water' OR 'drinking water' OR 'ground water' OR } \\
\text { 'treated water' }\end{array}$ \\
\hline 2. 'heavy metals' OR 'pesticides' OR 'microbial' \\
\hline 3. 'pollutants' OR 'contaminants' \\
\hline 4. 'Malaysia' \\
\hline 5. 'quality' \\
\hline 6. Combination of 1 AND 2 AND 3 AND 4 AND 5 \\
\hline
\end{tabular}

The search for potentially included articles was mainly through on-line bibliographic databases. Systematic searches were conducted using major relevant databases i.e. Science Direct, PubMed and Scopus using the keywords and search strategy (Table 1). In addition, Google Scholars were also used in order to capture articles which were unindexed. We also checked the references list of identified studies for potential included articles. The citation of potential eligible studies retrieved was exported into the citation manager (EndNote) and duplicates were eliminated. Two reviewers independently examined the abstracts for the inclusion criteria using the pre-designed data extraction form for eligibility (Appendix 1). If there were uncertainty or disagreement, the opinion of the third person was sought to resolve and decisions were made upon reading the full text article.

The quality of each included studies was assessed based on the following domains:

i) Optimal number of samples to determine the power of study

ii) Sample replication to ensure precision and accuracy of the results

iii) Water sampling method must follow the established methods

iv) Laboratory analysis replication to ensure consistency of the measurement

v) Quality control parameters which emphasize on Certified Reference Materials (CRM) for elementals and chemicals. Assessment risk of bias was evaluated using a form (Appendix 2).

If the elements of quality assessment were not mentioned anywhere in the articles, the risk of bias was considered high while scanty description on the elements were considered unclear. The risk of bias was considered low if the elements of quality assessment were described in detail.

\section{Statistical analysis}

The mean, standard deviation and number of samples for each parameters from the included studies were extracted from the articles. The mean values are then converted to weighted arithmetic mean. Articles with high weight contribute more to the weighted mean than articles with low weight. This is to differentiate the contribution of each article based on the number of samples collected. The overall weighted mean and 95\% confidence interval of all the parameters was then calculated to present the status of drinking water in Malaysia.

\section{Results}

\section{Search strategy results}

A total of 620 articles were discovered and 99 articles were identified through hand search and back searching from reference list. After screening for duplicates and relevant articles, 655 articles were excluded. The remaining 64 articles were assessed for eligibility criteria and (34) were excluded due to no relevant data available $(n=30)$ and review articles $(n=4)$. Thus, (30) articles were included in this review (Figure 1). No articles other than Malay and English language were found when the search strategy was conducted.

As mentioned in Figure 1, 29 articles were excluded in this review. Even though these articles were published after the year 2000, majority (86.2\%) were excluded since they were intervention studies. The remaining 4 articles were not included as they were proceedings and review article (Table 2). 
Table 2: Characteristics of studies included in the review.

\begin{tabular}{|c|c|c|c|c|c|c|}
\hline $\begin{array}{l}\text { Study No. as } \\
\text { Cited in Refer- } \\
\text { ence }\end{array}$ & $\begin{array}{l}\text { Study Abbre- } \\
\text { viation }\end{array}$ & $\begin{array}{l}\text { Study Loca- } \\
\text { tions }\end{array}$ & Water Sample & $\begin{array}{l}\text { Sampling } \\
\text { Date }\end{array}$ & $\begin{array}{l}{ }^{*} \text { Study } \\
\text { Design }\end{array}$ & $\begin{array}{c}\text { Water Quality Parameters Analyzed (Phys- } \\
\text { ical Parameters, Microbiology, Chemicals \& } \\
\text { Elements) }\end{array}$ \\
\hline [8] & $\begin{array}{l}\text { Ab Razak et } \\
\text { al. } 2016\end{array}$ & $\begin{array}{l}\text { Pasir Mas, } \\
\text { Kelantan }\end{array}$ & $\begin{array}{l}214 \text { drinking water samples } \\
\text { from residential }\end{array}$ & Not stated & $\mathrm{CS}$ & $\mathrm{Al}, \mathrm{Pb}, \mathrm{Fe}, \mathrm{Cu}, \mathrm{Cd}, \mathrm{Cr}, \mathrm{Ni}, \mathrm{Zn}$ \\
\hline [9] & $\begin{array}{l}\text { Abdullah \& } \\
\text { Soh } 2011\end{array}$ & $\begin{array}{c}\text { Peninsular } \\
\text { Malaysia (PM) }\end{array}$ & $\begin{array}{l}\text { Water treatment plant - TPO, } \\
\text { SRO, AOP }\end{array}$ & $\begin{array}{l}\text { June 2003- } \\
\text { May } 2004\end{array}$ & $\mathrm{CS}$ & $\begin{array}{c}\text { Alkenes (cis \& trans 1,2-DCE, TCE) } \\
\text { Alkanes (CHCl3, CHCl2Br, CHClBr2, 1,2-DBA) } \\
\text {-Aromatics (benzene, toluene, ethyl benzene, } \\
\text { chlorobenzene, 1,4 -DCB, 1,2-DCB) }\end{array}$ \\
\hline$[10]$ & $\begin{array}{l}\text { Abdullah et } \\
\text { al. } 2003\end{array}$ & $\begin{array}{l}\text { Tampin, Negeri } \\
\text { Sembilan \& } \\
\text { Sabak Bernam } \\
\text { Selangor } \\
\text { district }\end{array}$ & $\begin{array}{l}\text { Water treatment plant - TPO, } \\
\text { SRO, AOP }\end{array}$ & $\begin{array}{l}\text { The whole } \\
\text { year } 2001\end{array}$ & CS & Trihalomethanes (THM) \\
\hline$[11]$ & $\begin{array}{l}\text { Abu Hassan } \\
\text { et al. } 2011\end{array}$ & $\begin{array}{l}\text { Labu \& Langat } \\
\text { rivers }\end{array}$ & Water treatment plant - TPO & $\begin{array}{l}\text { January } \\
\text { 2005-Decem- } \\
\text { ber } 2009\end{array}$ & $\mathrm{CS}$ & Ammonia, manganese \\
\hline$[12]$ & $\begin{array}{l}\text { Ahmad et al. } \\
2015\end{array}$ & $\begin{array}{l}\text { Sungai Petani, } \\
\text { Kedah }\end{array}$ & $\begin{array}{c}9 \text { TW samples and } 3 \text { well } \\
\text { water samples collected from } \\
\text { different locations around } \\
\text { Sungai Petani, Kedah }\end{array}$ & Not stated & $\mathrm{CS}$ & As, $\mathrm{Cr}, \mathrm{Ni}, \mathrm{Cd}, \mathrm{Pb}$ \\
\hline$[13]$ & $\begin{array}{l}\text { Anita Devi et } \\
\text { al. } 2015\end{array}$ & $\begin{array}{l}\text { Petaling Jaya, } \\
\text { Selangor }\end{array}$ & $\begin{array}{l}100 \text { drinking water samples } \\
\text { from residentials and restau- } \\
\text { rants }\end{array}$ & Not stated & $\mathrm{CS}$ & $\begin{array}{l}\mathrm{pH} \text {, dissolved oxygen, biochemical oxygen de- } \\
\text { mand, temperature, turbidity, nitrate, phosphate, } \\
\text { total coliform }\end{array}$ \\
\hline$[4]$ & $\begin{array}{l}\text { Azrina et al. } \\
2011\end{array}$ & PM & $\begin{array}{l}\text { TW collected from } 2 \text { locations } \\
\text { from } 12 \text { states in PM }\end{array}$ & Not stated & $\mathrm{CS}$ & $\begin{array}{l}\text { Flouride, chloride, nitrate, suplhate, } \mathrm{Na}, \mathrm{Mg}, \mathrm{K}, \mathrm{Ca} \text {, } \\
\qquad \mathrm{Fe}, \mathrm{Cu}, \mathrm{Zn}, \mathrm{Cr}, \mathrm{Mn}, \mathrm{Ni}, \mathrm{As}, \mathrm{Cd}, \mathrm{Pb}\end{array}$ \\
\hline$[14]$ & $\begin{array}{l}\text { Azrina et al. } \\
\quad 2012\end{array}$ & PM & $\begin{array}{c}24 \text { bottles of DW and MW } \\
\text { samples were from } 22 \text { brands } \\
\text { collected from supermarkets } \\
\& \text { hypermarkets in Klang } \\
\text { Valley. The TW samples were } \\
\text { collected from different } \\
\text { locations in the } 12 \text { selected } \\
\text { states of PM }\end{array}$ & Not stated & $\mathrm{CS}$ & $\begin{array}{c}\mathrm{NO}_{3}^{--}, \mathrm{SO}_{4}^{2-}, \mathrm{Na}, \mathrm{Mg}, \mathrm{K}, \mathrm{Ca}, \mathrm{Fe}, \mathrm{Cu}, \mathrm{Zn}, \mathrm{Cr}, \mathrm{Mn}, \mathrm{Ni}, \mathrm{As}, \\
\mathrm{Cd}, \mathrm{Pb}, \mathrm{F}, \mathrm{Cl}\end{array}$ \\
\hline$[15]$ & $\begin{array}{l}\text { Chan et al. } \\
2007\end{array}$ & Klang Valley & $\begin{array}{l}\text { Filtered and unfiltered water } \\
\text { at point of use in houses } \\
\text { around Kajang, Serdang, Cy- } \\
\text { berjaya, Puchong and Bangi }\end{array}$ & Jul-04 & CS & $\begin{array}{l}\text { Turbidity, pH, Total suspended solid, Total coli- } \\
\text { form, E. coli, S. faecalis, P. aeruginosa }\end{array}$ \\
\hline$[16]$ & $\begin{array}{l}\text { Dzulfakar et } \\
\text { al. } 2011\end{array}$ & $\begin{array}{l}\text { Sg Lembing } \\
\text { \& Bukit Ubi } \\
\text { in Kuantan } \\
\text { District }\end{array}$ & $\begin{array}{l}\text { Collected from } 100 \text { respon- } \\
\text { dent kitchens' tap }\end{array}$ & Not stated & $\mathrm{CS}$ & $\mathrm{Al}$ \\
\hline$[17]$ & $\begin{array}{l}\text { Idrus et al. } \\
2014\end{array}$ & $\begin{array}{l}\text { Rural area, } \\
\text { Kelantan }\end{array}$ & $\begin{array}{l}\text { A total of } 454 \text { groundwater } \\
\text { samples were collected as } \\
\text { part of monitoring quality of } \\
\text { Water Supply and Environ- } \\
\text { mental Sanitation Program } \\
\text { (BAKAS). }\end{array}$ & $\begin{array}{l}\text { Mac - Dec } \\
2013\end{array}$ & $\mathrm{CS}^{*}$ & $\begin{array}{c}\text { Turbidity, } \mathrm{pH} \text {, total suspended solid, total coliform, } \\
\text { E. coli, S. faecalis, P. aeruginosa }\end{array}$ \\
\hline$[18]$ & Isa et al. 2014 & $\begin{array}{l}\text { Kapas Island, } \\
\text { Terengganu }\end{array}$ & $\begin{array}{l}126 \text { groundwater samples } \\
\text { collected from } 7 \text { boreholes } \\
\text { that were constructed per- } \\
\text { pendicular to the coastal area } \\
\text { with a maximum distance of } \\
\text { approximately } 150 \text { m from the } \\
\text { coastline. Borehole installa- } \\
\text { tions were done with different } \\
\text { depths }\end{array}$ & $\begin{array}{l}\text { Feb - April } \\
2011\end{array}$ & CS & $\mathrm{Ca}, \mathrm{Mg}, \mathrm{Na}, \mathrm{K}, \mathrm{HCO}_{3}, \mathrm{Cl}, \mathrm{SO}_{4}{ }^{2-}, \mathrm{Mn}, \mathrm{Cr}, \mathrm{Zn}, \mathrm{As}, \mathrm{Pb}, \mathrm{Cu}$ \\
\hline
\end{tabular}




\section{International Journal of Environmental Sciences \& Natural Resources}

\begin{tabular}{|c|c|c|c|c|c|c|}
\hline [19] & $\begin{array}{l}\text { Khoo et al } \\
2011\end{array}$ & $\mathrm{PM}$ & $\begin{array}{c}51 \text { samples of DW which } \\
\text { comprises of ROW (14), MW } \\
\text { (13) and TW (24) }\end{array}$ & $\begin{array}{l}\text { May - June } \\
2009\end{array}$ & $\mathrm{CS}$ & $\mathrm{Fe}, \mathrm{Cu}, \mathrm{Zn}, \mathrm{Pb}, \mathrm{Mn}$ \\
\hline \multirow[b]{2}{*}[20]{} & \multirow[b]{2}{*}{$\begin{array}{l}\text { Kumar et al. } \\
2014\end{array}$} & $\begin{array}{l}\text { Petaling Jaya \& } \\
\text { Puchong }\end{array}$ & \multirow{2}{*}{$\begin{array}{c}28 \text { environmental water and } \\
5 \text { DW samples were collected } \\
\text { in Malaysia; } 26 \text { environmental } \\
\text { water, } 3 \text { DW and } 2 \text { effluent } \\
\text { water were collected in } \\
\text { Thailand }\end{array}$} & $\begin{array}{l}\text { Oct- Dec } \\
2011\end{array}$ & $\mathrm{CS}$ & $\begin{array}{l}\text { Temperature, turbidity, salinity, pH, DO, total } \\
\text { dissolved solid }\end{array}$ \\
\hline & & $\begin{array}{l}\text { Songkla, the } \\
\text { Southeastern } \\
\text { coast of Thai- } \\
\text { land }\end{array}$ & & June 2012 & CS & $\begin{array}{l}\text { Giardia, Cryptosporidium Hookworm Ascaris, } \\
\text { Schistosoma, Blastocystiscysts, Entamoeba cysts, } \\
\text { Enterobius ova, Hymenolepis ova, tapeworm ova, } \\
\text { Toxocara ova, Toxoplasma oocysts, ectoparasites } \\
\text { of the orders Anastroca \& Cladocera, copepods, } \\
\text { lice, \& Brachinecta organisms }\end{array}$ \\
\hline$[21]$ & $\begin{array}{l}\text { Lim et al. } \\
2013\end{array}$ & $\begin{array}{l}\text { Seri Kemban- } \\
\text { gan, Selangor }\end{array}$ & $\begin{array}{l}100 \text { water samples were } \\
\text { collected from Taman Serdang } \\
\text { Raya, Taman Serdang Jaya, } \\
\text { Taman Sri Serdang, Taman } \\
\text { Universiti Indah, Taman Sun- } \\
\text { gai Besi Indah, Taman Belimb- } \\
\text { ing Indah, Taman Muhibbah, } \\
\text { Kampung Baru Seri Kemban- } \\
\text { gan \& Taman Bukit Serdang }\end{array}$ & $\begin{array}{l}21 \text { Dec } 2010 \\
-9 \text { Feb } 2011\end{array}$ & $\mathrm{CS}$ & $\begin{array}{l}\text { Temperature, } \mathrm{pH} \text {, conductivity, total dissolved } \\
\text { solid, } \mathrm{Pb}\end{array}$ \\
\hline$[22]$ & $\begin{array}{l}\text { Mohd Hasni } \\
\text { et al. } 2017\end{array}$ & Klang Valley & $\begin{array}{l}13 \text { samples from eight natural } \\
\text { spring water and five fla- } \\
\text { voured bottled drinking water }\end{array}$ & 2014 & $\mathrm{CS}$ & $\mathrm{Pb}, \mathrm{Cd}$ \\
\hline$[23]$ & $\begin{array}{l}\text { Mohd Sham } \\
\text { et al. } 2009\end{array}$ & $\begin{array}{c}\text { Selangor, } \\
\text { Perak, Melaka, } \\
\text { Kelantan, } \\
\text { Terengganu, } \\
\text { Kuala Lumpur } \\
\text { \& Kota Kinaba- } \\
\text { lu }\end{array}$ & $\begin{array}{c}3 \text { sites in Selangor \& } 1 \\
\text { site each in Perak, Melaka, } \\
\text { Kelantan, Terengganu, Kuala } \\
\text { Lumpur \& Kota Kinabalu. } \\
2067 \text { water samples were } \\
\text { collected from } 689 \text { selected } \\
\text { households }\end{array}$ & Not stated & $\mathrm{CS}^{*}$ & Fluoride \\
\hline$[24]$ & $\begin{array}{l}\text { Nalatambi } \\
2009\end{array}$ & $\begin{array}{l}\text { Bandar } \\
\text { Sunway Res- } \\
\text { idential area, } \\
\text { Petaling Jaya } \\
\text { Selangor }\end{array}$ & $\begin{array}{l}\text { Collected from four different } \\
\text { areas: Sunway University Col- } \\
\text { lege, Sunway Villa Apartment, } \\
\text { Sunway Condominium and } \\
\text { PJS 7/11 Bandar Sunway. }\end{array}$ & Not stated & $\mathrm{CS}$ & $\mathrm{Cu}, \mathrm{Cr}, \mathrm{Cd}, \mathrm{Zn}, \mathrm{Pb}, \mathrm{Ca}, \mathrm{Mg}$ \\
\hline [7] & $\begin{array}{l}\text { Ong et al. } \\
2007\end{array}$ & Klang Valley & $\begin{array}{c}\text { TW around S1-4, Sunway, } \\
\text { Subang Jaya, USJ, Puchong, Sri } \\
\text { Damansara, Damansara Jaya, } \\
\text { Tropicana, Bandar Utama, } \\
\text { Taman Tun, Bandsar, Section } \\
\text { 10, 12, 14, 17, } 19 \text { and PJ Old } \\
\text { Town }\end{array}$ & Not stated & $\mathrm{CS}$ & $\begin{array}{l}\text { Colour, } \mathrm{pH} \text {, turbidity, hardness, residual chlorine, } \\
\text { sulphate, } \mathrm{Zn}, \mathrm{Ba}, \mathrm{Cd}, \mathrm{Cr}, \mathrm{Pb}, \mathrm{Cu}, \mathrm{Fe}, \mathrm{Mn}, \mathrm{Mg}, \mathrm{Ni}\end{array}$ \\
\hline$[25]$ & $\begin{array}{l}\text { Praveena et } \\
\text { al. } 2018\end{array}$ & $\begin{array}{l}\text { Seri Kemban- } \\
\text { gan, Selangor }\end{array}$ & $\begin{array}{c}\text { Ninety water samples from } \\
\text { water vending machines in six } \\
\text { areas (Balakong, Serdang Jaya, } \\
\text { Seri Serdang, Taman Pinggiran } \\
\text { Putra, Taman Equine, Lestari } \\
\text { Perdana) }\end{array}$ & Not stated & $\mathrm{CS}^{*}$ & E. coli \\
\hline$[26]$ & $\begin{array}{l}\text { Qaiyum et al. } \\
\quad 2011\end{array}$ & $\begin{array}{l}\text { Mukim Parit } \\
\text { Lubok \& Parit } \\
\text { Raja, Batu } \\
\text { Pahat Johor } \\
\quad \text { Perak }\end{array}$ & $\begin{array}{l}\text { Drinking water samples were } \\
\text { collected at the } 100 \text { respon- } \\
\text { dent's kitchen tap. }\end{array}$ & Not stated & $\mathrm{CS}$ & $\mathrm{Al}$ \\
\hline
\end{tabular}




\section{International Journal of Environmental Sciences \& Natural Resources}

\begin{tabular}{|c|c|c|c|c|c|c|}
\hline [27] & $\begin{array}{l}\text { Rahmanian et } \\
\text { al. } 2015\end{array}$ & Perak & $\begin{array}{l}\text { Collected from ex-minerals } \\
\text { and mining activities in } \\
\text { Bandar Universiti, Bandar Seri } \\
\text { Iskandar, Siputeh, Tronoh, } \\
\text { Taman Maju, Batu Gajah, Uni- } \\
\text { versiti Teknologi Petronas \& } \\
\text { Ipoh. Drinking water samples } \\
\text { were collected from TW of } \\
\text { residential and commercial } \\
\text { areas while mineral and ROW } \\
\text { samples were purchased from } \\
\text { local supermarkets }\end{array}$ & Not stated & $\mathrm{CS}^{*}$ & $\begin{array}{l}\text { pH, conductivity, turbidity, } \mathrm{Cu}, \mathrm{Zn}, \mathrm{Mg}, \mathrm{Fe}, \mathrm{Cd}, \mathrm{Pb} \text {, } \\
\mathrm{Cr}, \mathrm{As}, \mathrm{Hg}, \mathrm{Sn}\end{array}$ \\
\hline [28] & $\begin{array}{l}\text { Richard et al. } \\
2016\end{array}$ & Sarawak & $\begin{array}{l}\text { Two drinking water treatment } \\
\text { plants and seven distribution } \\
\text { system sites }\end{array}$ & $\begin{array}{l}\text { July } 2012-0 c- \\
\text { tober } 2013\end{array}$ & CS & $\begin{array}{l}\text { pH, temperature, conductivity, total dissolved } \\
\text { solids, salinity, dissolved oxygen, turbidity, } \\
\text { ammonia, chlorine, nitrite, nitrate, fluoride, } \\
\text { parasites (Giardia, Cryptosporidium, Spirometra, } \\
\text { Blastocystis, nematode larvae-like, Taenia ova-like, } \\
\text { Acanthamoeba, Naegleria) }\end{array}$ \\
\hline [29] & $\begin{array}{l}\text { Santhi et al. } \\
\quad 2012\end{array}$ & $\begin{array}{l}\text { Langat River } \\
\text { basin }\end{array}$ & $\begin{array}{l}\text { River waters samples were } \\
\text { collected from Semenyih \& } \\
\text { Labu River, } 30 \text { TW samples } \\
\text { were collected from houses in } \\
\text { Kuala Lumpur \& } 9 \text { brands of } \\
\text { MW bottled in PET containers } \\
\text { were purchased from shops } \\
\text { around Kuala Lumpur }\end{array}$ & $\begin{array}{l}\text { Sept } 2008- \\
\text { March } 2009 .\end{array}$ & CS & $\begin{array}{c}\text { Turbidity, pH, temperature, Dissolved oxygen, } \\
\text { Bisphenol A }\end{array}$ \\
\hline$[30]$ & $\begin{array}{l}\text { Shaharudin } \\
\text { et al. 2010a }\end{array}$ & $\begin{array}{c}\text { Pasir Mas Ke- } \\
\text { lantan, Kuala } \\
\text { Terengganu, } \\
\text { Terengganu \& } \\
\text { Kota Kinabalu, } \\
\text { Sabah }\end{array}$ & $\begin{array}{l}\text { Three replicated water sam- } \\
\text { ples were collected from each } \\
\text { sampling site. A total of } 247 \text { of } \\
\text { parallel number of respon- } \\
\text { dents from each study site } \\
\text { involved in this study }\end{array}$ & Not stated & $\mathrm{CS}^{*}$ & Fluoride \\
\hline [31] & $\begin{array}{l}\text { Shaharuddin } \\
\text { et al. } 2010 \mathrm{~b}\end{array}$ & $\begin{array}{l}\text { Selangor, } \\
\text { Melaka, Perak, } \\
\text { Kelantan, } \\
\text { Kuala Lumpur, } \\
\text { Terengganu, } \\
\text { Sabah }\end{array}$ & $\begin{array}{l}2067 \text { water samples were } \\
\text { analysed for fluoride }\end{array}$ & Not stated & CS & Fluoride \\
\hline$[32]$ & $\begin{array}{l}\text { Siti Farizwa- } \\
\text { na et al. } 2010\end{array}$ & $\begin{array}{l}\text { Kota Tinggi, } \\
\text { Johor }\end{array}$ & $\begin{array}{l}6 \text { palm oil estates with private } \\
\text { water supply and } 4 \text { estates } \\
\text { with public water supply. }\end{array}$ & $\begin{array}{l}\text { Jan } 2009- \\
\text { Jan } 2010\end{array}$ & $\mathrm{CS}^{*}$ & $\begin{array}{l}\mathrm{pH} \text {, total dissolved solid, turbidity, residual chlo- } \\
\text { rine, } \mathrm{Al}\end{array}$ \\
\hline [33] & $\begin{array}{l}\text { Soh \& Abdul- } \\
\quad \text { lah } 2007\end{array}$ & PM & $\begin{array}{l}\text { Water treatment plant - TPO, } \\
\text { SRO, AOP }\end{array}$ & $\begin{array}{l}\text { June } 2003- \\
\text { May } 2004\end{array}$ & CS & $\begin{array}{l}\text { Trichloroethylene, } \mathrm{CHCl}_{3}, \mathrm{CHCl}_{2} \mathrm{Br}, \mathrm{CHClBr}_{2} \text {, total } \\
\text { trihalomethane, benzene, toluene, ethyl benzene, } \\
\text { chlorobenzene, 1,4 -DCB, 1,2-DCB }\end{array}$ \\
\hline [34] & $\begin{array}{l}\text { Tan et al. } \\
2016\end{array}$ & Kelantan & $\begin{array}{l}\text { Seventeen water samples } \\
\text { from water vending machines } \\
\text { in three localities in Kelantan }\end{array}$ & Not stated & $\mathrm{CS}$ & $\mathrm{pH}$, temperature, turbidity, E. coli \\
\hline [35] & $\begin{array}{l}\text { Yusof et al. } \\
2001\end{array}$ & Johor & $\begin{array}{l}\text { Disused mining pool through- } \\
\text { out PM and } 27 \text { WTP in Johor } \\
\text { state }\end{array}$ & Not stated & $\mathrm{CS}$ & $\begin{array}{c}\mathrm{pH} \text {, temperature, radionuclides (radium, thorium, } \\
\text { uranium) }\end{array}$ \\
\hline
\end{tabular}

Cross sectional descriptive (CS), CS*-Cross sectional with emphasis on risk assessment; TPO-treatment plant outlet, SRO-service reservoir outlet, AOP-auxiliary outlet point; DW-drinking water, MW-mineral water, TW-tap water, ROW-reverse osmosis water.

\section{Study locations}

Of the thirty papers included in the review, nine studies were conducted in Peninsular Malaysia consisting of several states $[4,9,14,19,23,30,31,33,35]$. A total of eighteen studies focused their study locations in a single state namely Selangor
[7,13,15,21,22,24,25,29], Kedah [12], Pahang [16], Kelantan [8,17,34], Terengganu [18], Johor [26,32], Perak [27] and Sarawak [28]. Two studies were conducted in selected areas in both Selangor \& Negeri Sembilan $[10,11]$, two studies were conducted in more than five states namely Selangor, Perak, Melaka, Kelantan, Terengganu, Kuala Lumpur and Sabah [23] and one study was conducted in three states which were Kelantan, Terengganu and 
Sabah [30]. In addition, one study was conducted in collaboration with the neighbouring country, Thailand as well as Selangor [20]; (it is worthy to note that this review only considers data for Malaysia and do not include those from Thailand).

\section{Water samples}

Majority of the studies collected water samples from domestic kitchen tap $[4,7,8,12-16,19,21,23,24,26,27,29-32]$ while only few studies focused on drinking water treatment plant (DWTP) samples [9,10,28,33,35]. At least three studies [11,14,22,27,29] bought some of their samples from selected supermarket or hypermarket situated around their study areas. Water samples also comprised well water [12] as well as ground water samples $[17,18]$. In addition, two studies collected samples from water vending machines in Selangor and Kelantan [25,34].

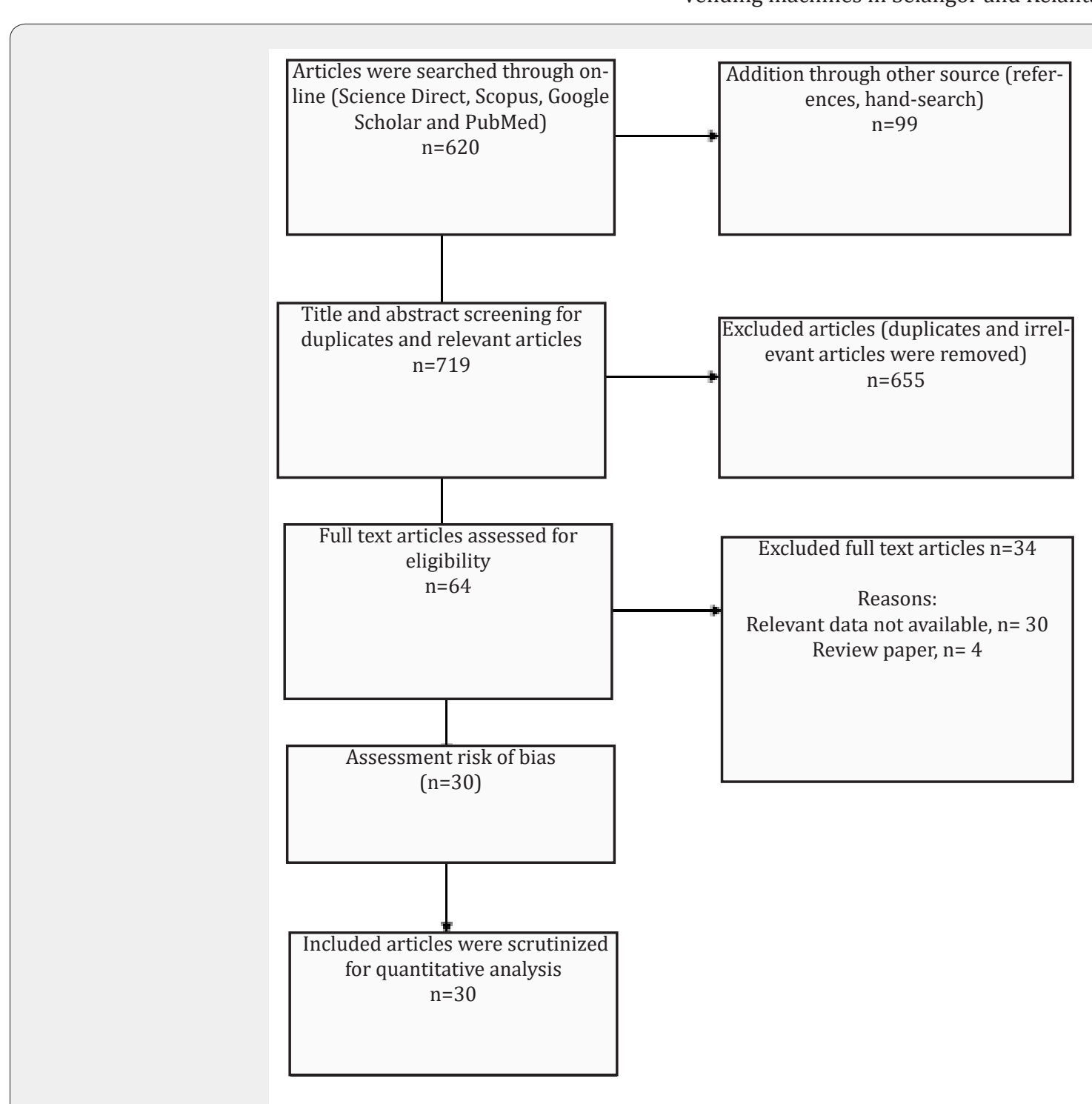

Figure 1: Flowchart for search strategy results.

\section{Sampling date}

The earliest drinking water samples were collected in 2001 and the latest were in 2014. However, half of the selected studies (thirteen studies) did not mention sampling date. Five studies were conducted within one-year period [9,10,29,32-33]. Six studies completed their sample collection within 1-3 months' time $[15,18-21,28]$. Only one study analysed water samples in a long run, however these researchers used secondary data [11].

\section{Study design}

Cross sectional descriptive (CS) dominated studies conducted on drinking water quality in Malaysia followed by six studies with emphasis on risk assessment to human health. 


\section{Water quality parameters}

The search resulted in 64 studies retained for full text review. In total, 30 studies met the inclusion criteria. Ten studies reported on physical parameters including $\mathrm{pH}$, temperature, turbidity, conductivity, total suspended solid (TSS), total dissolved solid (TDS), dissolved oxygen (DO), colour and water hardness in water samples originating from water treatment plants (WTP), tap water, mineral water, bottled drinking water as well as water from vending machines. Nineteen studies reported on elements in water samples such as arsenic (As), cadmium (Cd), lead $(\mathrm{Pb})$, manganese $(\mathrm{Mn})$, copper $(\mathrm{Cu})$, chromium $(\mathrm{Cr})$, iron $(\mathrm{Fe})$, sodium $(\mathrm{Na})$, potassium $(\mathrm{K})$, zinc $(\mathrm{Zn})$, nickel $(\mathrm{Ni})$, magnesium $(\mathrm{Mg})$, calcium $(\mathrm{Ca})$, aluminium $(\mathrm{Al})$, mercury $(\mathrm{Hg})$, stanum $(\mathrm{Sn})$, barium $(\mathrm{Ba})$, fluoride $\left(\mathrm{F}^{-}\right)$, nitrate $\left(\mathrm{NO}_{3}{ }^{-}\right)$and sulphate $\left(\mathrm{SO}_{4}{ }^{2-}\right.$ ). One study reported on radionuclides (radium, uranium and thorium) in disused mining pool. Six studies measured chemical parameters such as trihalomethane (THM), bisphenol A (BPA), residual chlorine and ammonia. Two studies focused on volatile organic compounds (VOCs) in WTP such as Trichloroethylene, $\mathrm{CHCl}_{3}, \mathrm{CHCl}_{2} \mathrm{Br}, \mathrm{CHClBr}_{2}$, total trihalomethene, benzene, toluene, ethyl benzene, chlorobenzene, 1,4 -DCB, 1,2-DCB, alkenes (cis \& trans 1,2-DCE, TCE), alkanes $\left(\mathrm{CHCl}_{3}, \mathrm{CHCl}_{2} \mathrm{Br}, \mathrm{CHClBr}_{2}\right.$, 1,2-DBA) and aromatics (benzene, toluene, ethyl benzene, chlorobenzene, 1,4 -DCB, 1,2-DCB). Four studies described on microbiological aspects which include total coliform, faecal coliform, E.coli, S. faecalis, P. aeruginosa in drinking water, WTP and water from vending machines. One study reported on Giardia, Cryptosporidium, Hookworm Ascaris, Schistosoma, Blastocystis cysts, Entamoebacysts, Enterobius ova, Hymenolepis ova, tapeworm ova, Toxocara ova, Toxoplasma oocysts, ectoparasites of the orders Anastroca and Cladocera, copepods, lice and Brachinecta organisms in environmental, drinking and effluent water samples.

\section{Study quality}

More than three quarter of included articles were published in peer-reviewed journal.

\section{Quality assessment}

Table 3 reports on the overall quality assessment of parameters analysed for drinking water quality for the included articles in this review while Table 4 shows its summary. Out of 35 articles included in this review, two articles $[8,28]$ fulfilled requirement for four quality assessment parameters (Table 3 ). Meanwhile, two articles $[4,17,25]$ fulfilled requirement for three quality assessment parameters. Nine articles met only two criteria of quality assessment parameters. About four articles did not fulfil the requirement of quality assessment, where two articles did not properly described their sampling methods $[11,23]$ while another two articles did not include any quality assessment parameters in the methodology of the article $[10,13]$. More than $50 \%$ of the articles were at high risk of bias (56.0\%), while only $30.7 \%$ of the quality assessment parameters showed low risk of bias (Table 3). The remaining $22.2 \%$ of the quality assessment of included articles did not describe the quality assessment clearly. When individually analyzing each item of the quality assessment parameter, it can be noted that nearly three quarter (73.3\%) of the articles reported a clear and well defined sampling methods while about $33.3 \%$ of articles reported on sample replication and another $23.3 \%$ of articles reported proper quality control parameters. A total of $16.7 \%$ of articles reported on analysis replication while only two studies $[8,17]$ calculated their sample sizes (Table 4).

Table 3: Study quality and risk of bias.

\begin{tabular}{|c|c|c|c|c|c|c|c|}
\hline No & References & $\begin{array}{l}\text { Sample Size } \\
\text { Calculation }\end{array}$ & $\begin{array}{l}\text { Sample Rep- } \\
\text { lication }\end{array}$ & $\begin{array}{l}\text { Sampling } \\
\text { Method }\end{array}$ & $\begin{array}{c}\text { Analysis } \\
\text { Replication }\end{array}$ & $\begin{array}{l}\text { Quality } \\
\text { Control }\end{array}$ & $\begin{array}{c}\text { Total Low Risk of } \\
\text { Bias }\end{array}$ \\
\hline 1. & Ab Razak et al. [8] & $\mathbf{0}$ & $\mathbf{0}$ & $\mathbf{0}$ & ○ & $\mathbf{0}$ & 4 \\
\hline 2. & Richard et al. [28] & 0 & $\mathbf{0}$ & $\mathbf{0}$ & $\mathbf{0}$ & $\mathbf{0}$ & 4 \\
\hline 3. & Azrina et al. [14] & 0 & $\mathbf{0}$ & $\mathbf{0}$ & $\emptyset$ & $\mathbf{0}$ & 3 \\
\hline 4. & Idrus et al. [17] & $\mathbf{0}$ & 0 & $\mathbf{0}$ & $\mathbf{0}$ & ○ & 3 \\
\hline 5. & Praveena et al. [25] & ○ & $\mathbf{0}$ & $\mathbf{0}$ & $\mathbf{0}$ & $\varnothing$ & 3 \\
\hline
\end{tabular}




\begin{tabular}{|c|c|c|c|c|c|c|c|}
\hline 6. & Abdullah \& Soh [9] & $\bullet$ & $\bullet$ & $\mathbf{0}$ & $\bullet$ & $\mathbf{0}$ & 2 \\
\hline 7. & Azrina et al. [4] & - & 0 & $\emptyset$ & $\emptyset$ & $\mathbf{0}$ & 2 \\
\hline 8. & Chan et al. [15] & $\bullet$ & $\bullet$ & $\mathbf{0}$ & $\mathbf{0}$ & $\bullet$ & 2 \\
\hline 9. & Dzulfakar et al. [16] & • & 0 & $\mathbf{0}$ & $\emptyset$ & $\emptyset$ & 2 \\
\hline 10. & Isa et al. [18] & - & $\bullet$ & $\mathbf{0}$ & $\mathbf{0}$ & $\emptyset$ & 2 \\
\hline 11. & Khoo et al. [19] & • & $\mathbf{0}$ & $\mathbf{0}$ & ○ & $\emptyset$ & 3 \\
\hline 12. & Qaiyum et al. [26] & - & $\mathbf{0}$ & $\mathbf{0}$ & - & $\bullet$ & 2 \\
\hline 13. & Soh \& Abdullah [33] & - & $\bullet$ & $\mathbf{0}$ & - & $\mathbf{0}$ & 2 \\
\hline 14. & Yusof et al. [35] & $\bullet$ & $\bullet$ & $\mathbf{0}$ & - & $\mathbf{0}$ & 2 \\
\hline 15. & Ahmad et al. [12] & $\bullet$ & $\bullet$ & o & • & $\emptyset$ & 1 \\
\hline 16. & Kumar et al. [20] & $\bullet$ & $\bullet$ & $\mathbf{0}$ & $\emptyset$ & $\bullet$ & 1 \\
\hline 17. & Lim et al. [21] & - & ○ & $\mathbf{0}$ & - & $\emptyset$ & 1 \\
\hline 18. & Mohd Hasni et al. [22] & • & $\bullet$ & $\mathbf{0}$ & • & $\bullet$ & 1 \\
\hline 19. & Nalatambi [24] & $\bullet$ & 0 & $\bullet$ & $\emptyset$ & $\emptyset$ & 1 \\
\hline 20. & Ong et al. [7] & • & $\bullet$ & $\mathbf{0}$ & - & $\emptyset$ & 1 \\
\hline 21. & Rahmanian et al. [27] & $\bullet$ & $\bullet$ & $\mathbf{0}$ & - & $\bullet$ & 1 \\
\hline 22. & Santhi et al. [29] & $\bullet$ & $\bullet$ & $\mathbf{0}$ & - & $\emptyset$ & 1 \\
\hline 23. & Shaharuddin et al. [30] & $\bullet$ & $\bullet$ & $\mathbf{0}$ & • & $\bullet$ & 1 \\
\hline 24. & Siti Farizwana et al. [32] & $\bullet$ & $\bullet$ & $\mathbf{0}$ & - & $\emptyset$ & 1 \\
\hline 25. & Tan et al. [34] & $\bullet$ & $\bullet$ & $\mathbf{0}$ & - & $\emptyset$ & 1 \\
\hline 26. & Abdullah et al. [10] & $\bullet$ & $\bullet$ & $\bullet$ & - & $\bullet$ & $\mathbf{0}$ \\
\hline 27. & Abu Hasan et al. [11] & $\bullet$ & $\bullet$ & $\emptyset$ & • & $\bullet$ & $\mathbf{0}$ \\
\hline
\end{tabular}




\begin{tabular}{|c|c|c|c|c|c|c|}
\hline 28. & Anita Devi et al. [13] & $\bullet$ & $\bullet$ & $\bullet$ & $\bullet$ \\
\hline 29. & Mohd Sham et al. [23] & $\bullet$ & $\bullet$ & $\bullet$ & $\mathbf{0}$ \\
\hline 30. & Shaharuddin et al. [31] & $\bullet$ & $\bullet$ & $\mathbf{0}$ & $\mathbf{0}$ \\
\hline & Total low risk of bias & $\mathbf{2}$ & $\mathbf{1 0}$ & $\mathbf{2 2}$ & $\mathbf{5}$ & $\mathbf{7}$ \\
\hline
\end{tabular}

Key: $\bullet$ High risk of bias O Low risk of bias $\varnothing$ Unclear.

Table 4: Summary of quality assessment parameters score (\%) for included articles.

\begin{tabular}{|c|c|c|c|}
\hline Quality Assessment Parameters for included Articles & Low Risk of Bias & High Risk of Bias & Unclear \\
\hline Sample size calculation & 6.7 & 93.3 & none \\
\hline Sample replication & 33.3 & 13.3 & none \\
\hline Sampling method & 73.4 & 66.7 & 13.3 \\
\hline Analysis replication & 16.7 & 40 & 36.7 \\
\hline Quality Control & 23.3 & 56 & 22.2 \\
\hline Average score (\%) & 30.7 & 56.7 \\
\hline
\end{tabular}

\section{Results for physical parameter}

Figure 2 shows the pooled mean of physical parameters in reviewed articles which include $\mathrm{pH}$, turbidity, total suspended solid (TSS), total dissolved solid (TDS), and, temperature. There were twelve articles reporting on $\mathrm{pH}$, ten on turbidity, two on TSS, four on TDS, and seven on temperature. Reported pH levels from most of the studies complied with Malaysian National Drinking Water Quality Standard (NDWQS) except for studies by [15,32]. Study by [32] also demonstrated turbidity levels almost two times exceeding the 5 NTU limit in the NDWQS. The range of fluoride reported by both articles by [30,31] were below than the range stipulated in NDWQS. To date, no reference levels exist for TSS, temperature, sulphate, and fluorine.

\section{Results for total element concentrations}

Pooled average and range of total elements concentrations $(\mathrm{mg} / \mathrm{L})$ in drinking water in Malaysia as reported by included articles are presented in Figure 3-6. Figure 4 showed the pooled average and range of total element concentrations $(\mathrm{mg} / \mathrm{L})$ that exceeded the NDWQS; they were arsenic (As), cadmium (Cd), lead $(\mathrm{Pb})$, manganese $(\mathrm{Mn})$, nickel $(\mathrm{Ni})$, aluminium $(\mathrm{Al})$, chromium $(\mathrm{Cr})$ and, copper (Cu). Elements concentrations varied widely for different studies. The highest concentrations and the only exceeding average value for Mg were reported by [7] while for other studies all values were below the NDWQS of 150mg/L. Three studies reported on Al contamination in drinking water in this country, average results reported by [32] showed Al levels which were more than triple compared to the NDWQS while [26] reported average level of Al close to NDWQS value $(0.2 \mathrm{mg} / \mathrm{L})$. Out of three studies analyzing $\mathrm{Ni}$ in drinking water, only [4] reported levels more than the NDWQS of $0.02 \mathrm{mg} / \mathrm{L}$. They reported levels of more than fifty times higher than the NDWQS that may also dragged the overall value for $\mathrm{Ni}$ in drinking water. The condition is similar to as levels in drinking water; however, this element was reported by five studies. Again, [4] reported higher as content in their samples. For $\mathrm{Cd}$, out of six studies, half of them reported $\mathrm{Cd}$ levels higher the NDWQS of $0.01 \mathrm{mg} / \mathrm{L}$. This review managed to capture ten studies that reported on Pb levels in drinking water samples, and out of that, three studies reported higher than the NDWQS $(4,12,24)$ while one study at the borderline [7].

Figure 5 showed pooled average and range of total elements concentrations in drinking water within the NDWQS. Those elements were sodium ( $\mathrm{Na}$ ) and zinc $(\mathrm{Zn})$. Figure 6 showed two elements that has been detected in drinking water by few studies, but no levels were established for the NDWQS. These elements include potassium $(\mathrm{K})$ and calcium $(\mathrm{Ca})$.

\section{Chemical parameters}

In this review, chemical parameters include ammonia, trihalomethane (THM), chloroform, bisphenol A (BPA), trichloroethylene (TCE), benzene, toluene, ethylbenzene, chlorobenzene, dichlorobromomethane, and other volatile organic compounds (VOCs) such as 1,2 DBA, 1,4 DCB and 1,2 DCB which originated from four studies $[9,10,29,33]$. 

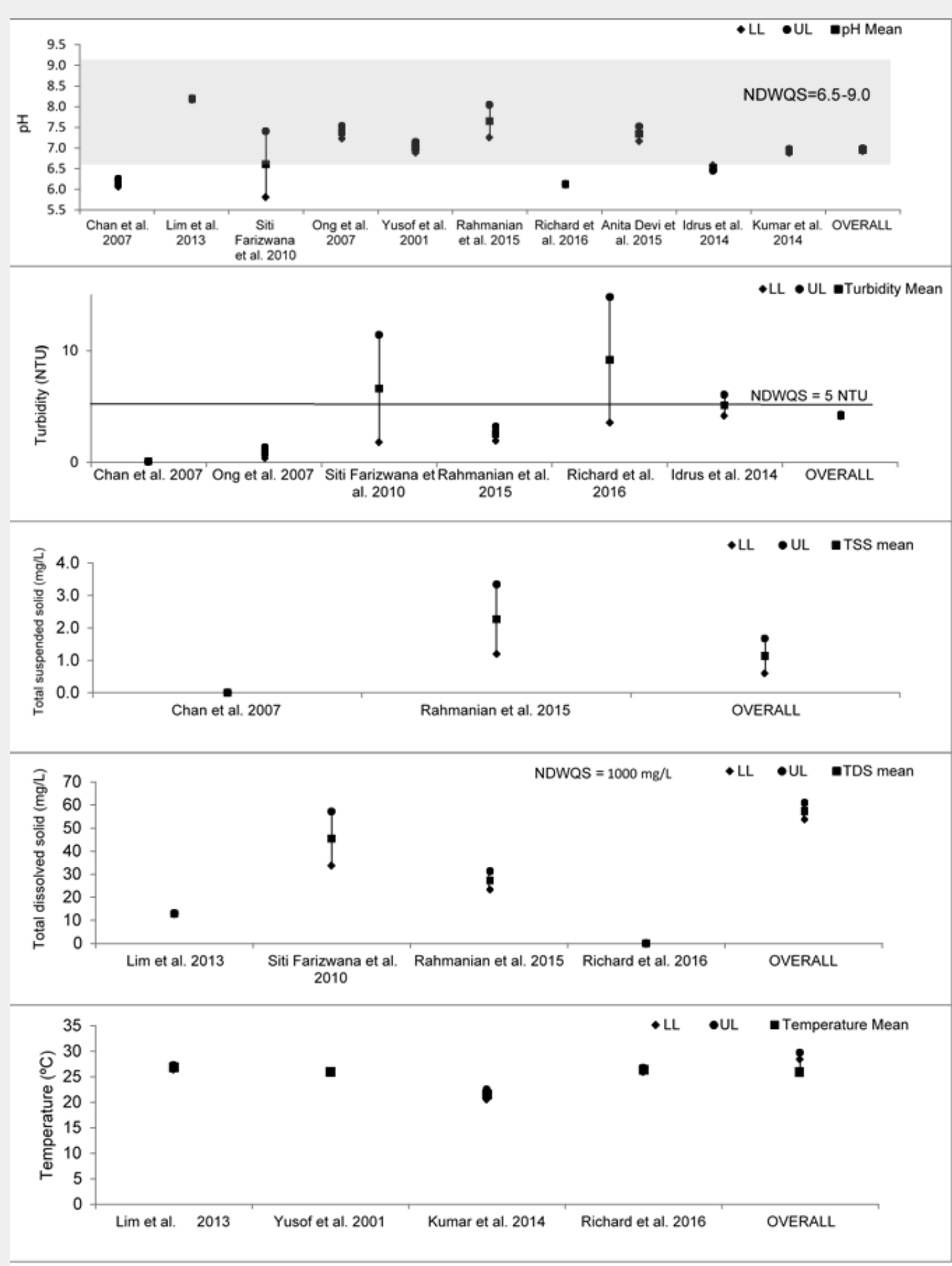

Figure 2: Pooled average and range of physical parameters levels in drinking water as reported by included articles.

Measurements of VOCs were reported by [9,33]. The highest eleven organic compounds at different sampling points in Negeri Sembilan including chlorobenzene, dibromochloromethane, toluene and 1,1,1,2-Tetrachloroethane were described by [33].
Similar VOCs were measured by [9] in a study conducted in Peninsular Malaysia. None of the VOC concentrations from these two studies exceeded the NDWQS. 


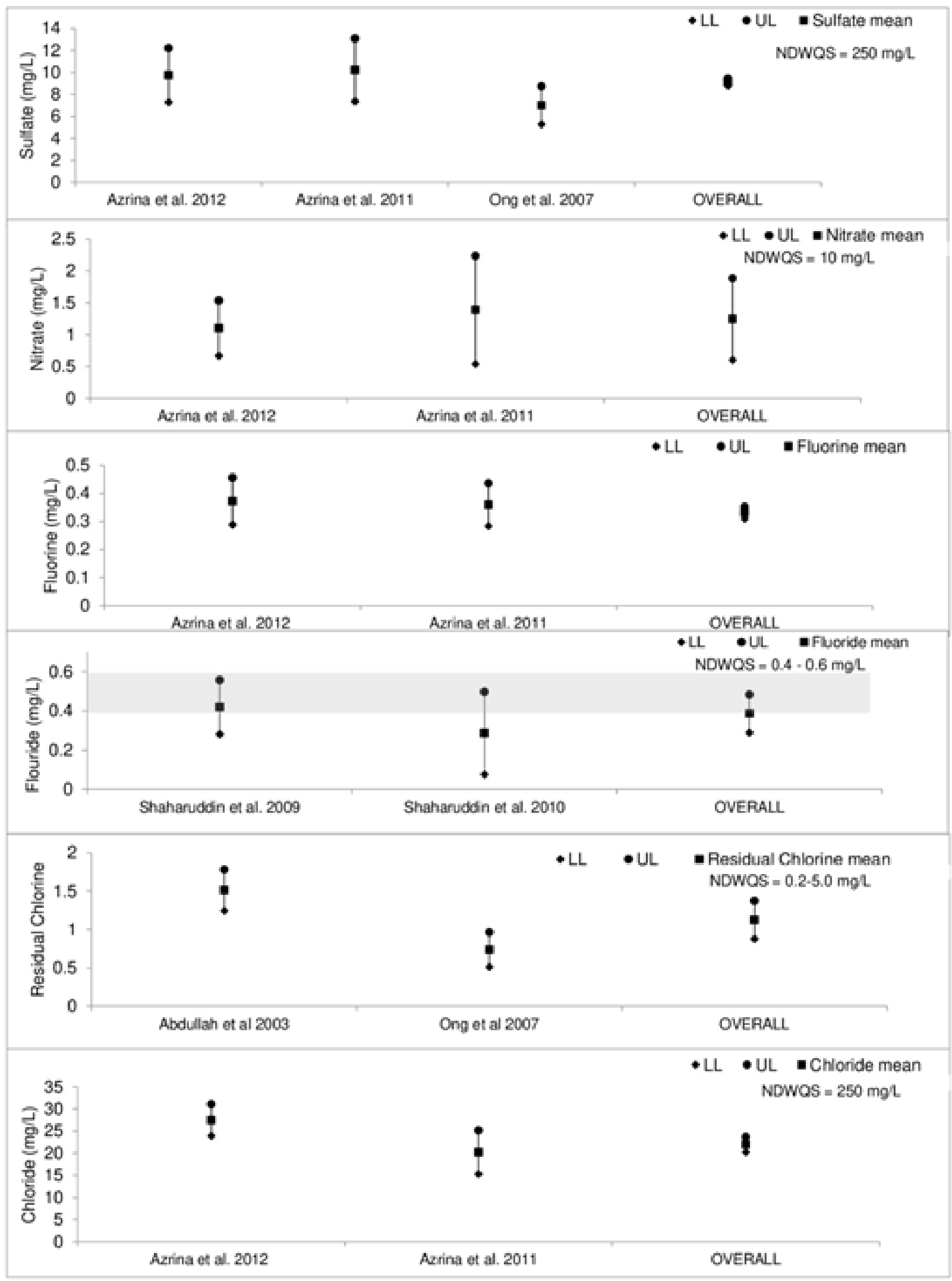

Figure 3: Pooled average and range of inorganic ion levels in drinking water as reported by included articles 


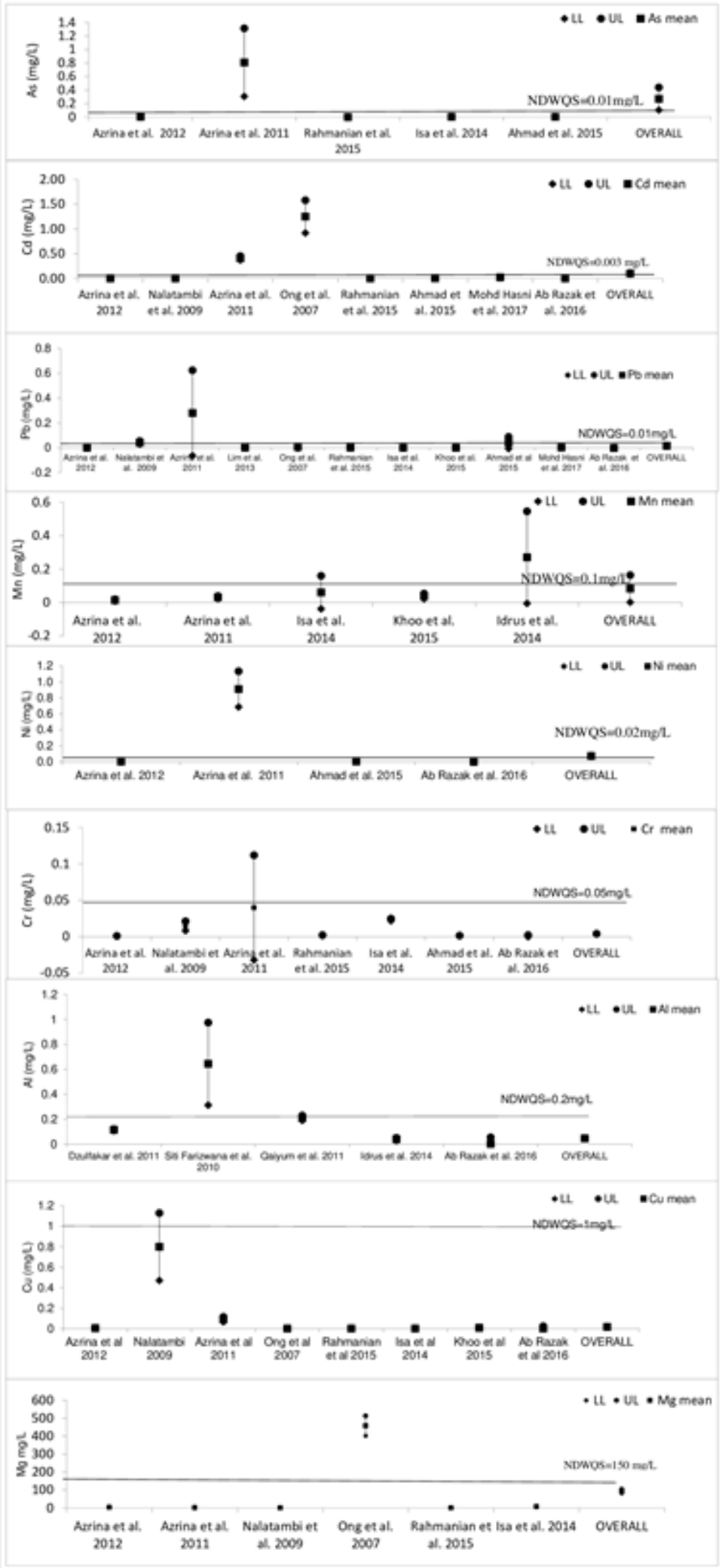

Figure 4: Pooled average and range of total elements concentrations $(\mathrm{mg} / \mathrm{L})$ in drinking water that exceeded NDWQS in Malaysia as reported by included articles. 


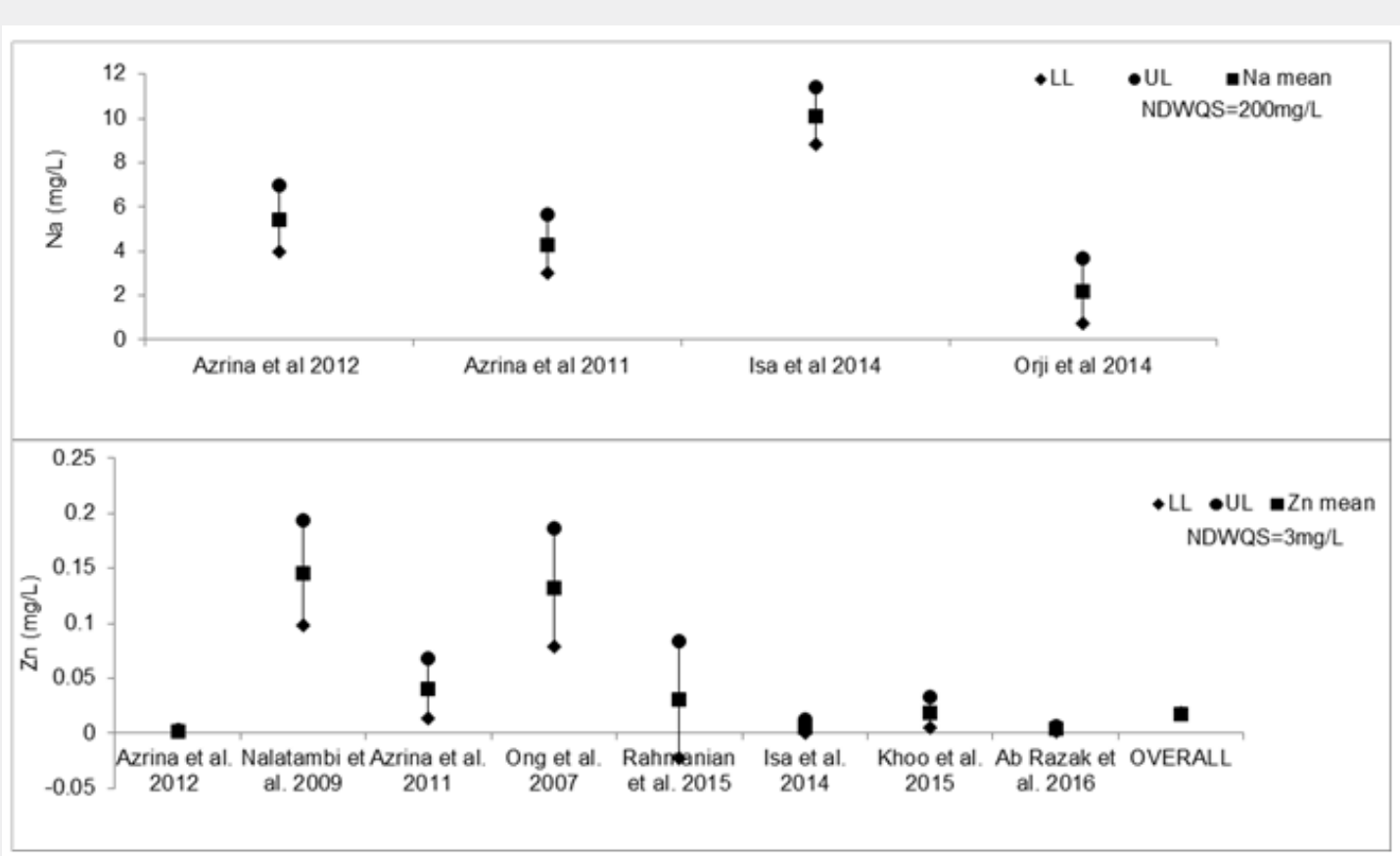

Figure 5: Pooled average and range of total elements concentrations ( $\mathrm{mg} / \mathrm{L})$ in drinking water that did not exceed the NDWQS in Malaysia as reported by included articles.

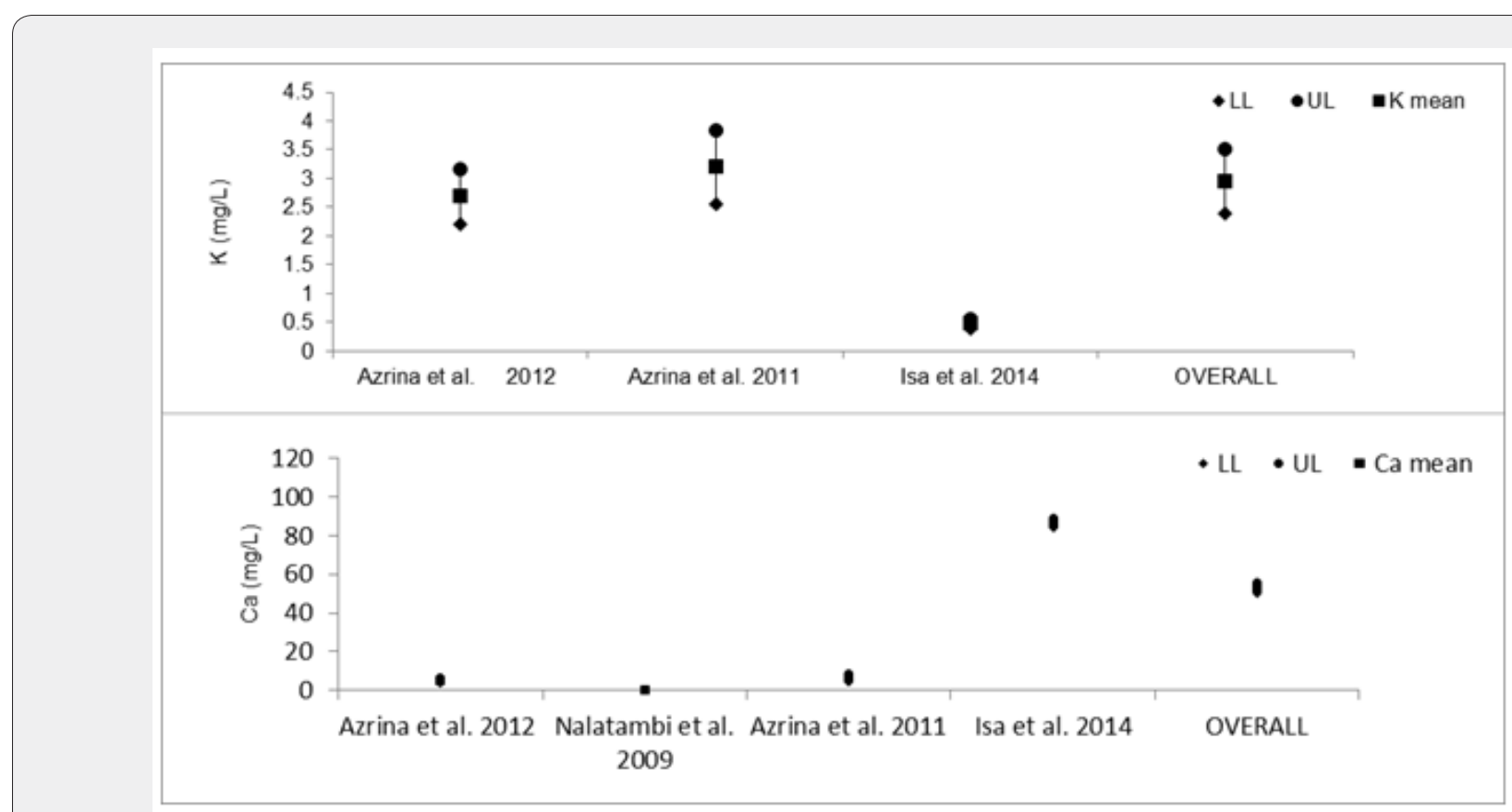

Figure 5: Pooled average and range of total elements concentrations (mg/L) in drinking water with no NDWQS values in Malaysia as reported by included articles

THM concentrations reported by [10] ranged from 2.4 to $136.5 \mu \mathrm{g} / \mathrm{L}$ (average: $61.0 \pm 18.0 \mu \mathrm{g} / \mathrm{L}$ ) from distribution system for districts of Tampin and Sabak Bernam. None of the samples exceeded the stipulated THM guidelines of $1000 \mu \mathrm{g} / \mathrm{L}$ in the NDWQS.
A study by [29] reported BPA concentrations in tap water and bottled polyethylene (PET) containers. BPA concentrations in 30 tap water samples ranged from 3.5 to $59.8 \mathrm{ng} / \mathrm{L}$ (average: $14.1 \pm 14.4 \mathrm{ng} / \mathrm{L}$ ) with highest BPA concentrations detected in samples connected to PVC pipes and water filter devices. Mean 
BPA concentrations in mineral bottled water at room temperature $\left(25^{\circ} \mathrm{C}\right)$ were significantly lower $(3.3 \pm 2.6 \mathrm{ng} / \mathrm{L})$ compared to those stored at $50^{\circ} \mathrm{C}(11.3 \pm 5.3 \mathrm{ng} / \mathrm{L})$. BPA is not listed under the NDWQS.

\section{Discussions}

This review aims to shed light on the status of drinking water quality in Malaysia. Thus far, numerous studies have been conducted pertaining to physical, elemental, chemical and microbiological properties in drinking water. After screening articles for inclusion criteria, only thirty articles were included in this review.

All the included articles were of cross-sectional study design. We constructed domains for quality assessment of such studies which consist of sample size calculation, sample replication, sampling method, analysis replication and quality control for the lab techniques. Those quality assessment parameters are crucial to ensure more reliable and valid results. With an exception of one article [17], majority of the included articles did not perform the calculation of optimum sample size needed. Sample size calculation and stratification of sampling point is necessary to enable the inference of the result [36]. Other than that, quality assessment criteria which was lacking by the researchers were replication of the analysis. Thus, it is possible that these studies may be subjected to systematic errors resulted from observer bias and information bias.

In the NDWQS, recommended level for $\mathrm{pH}$ is between 6.5 to 9. Most of the included articles were within the range except for study by [15,32]. Study by [32] reported pH levels in drinking water between 5.81 to 7.41 which were slightly acidic for some of the samples. As for turbidity, the level of 5 NTU stipulated in the NDWQS was also exceeded by [32]. Study by [32] was conducted in palm oil estates in Kota Tinggi, Johor which used water samples from private water supply. Inefficiency of water treatment system as well as lack of training by personnel who handled the water treatment process were the main reasons for violation of water samples for $\mathrm{pH}$ and turbidity. Study by [15] reported slightly acidic drinking water samples from Kajang, Cyberjaya and Bangi. However, reasons for violation were not mentioned in the study.

Physical parameters such as $\mathrm{pH}$ is an important parameter as low pH may cause corrosion of pipes resulting in the presence of certain heavy metals such as cadmium, copper, lead and zinc although there is no health-based guideline value [37]. The WHO Guidelines for Drinking Water Quality 2007 suggests pH level between 6.5 to 9.5 in comparison with NDWQS for $\mathrm{pH}$ between 6.5 to 9.0. Although the upper limit for $\mathrm{pH}$ in the NDWQS is slightly lower than the WHO guidelines, $\mathrm{pH}$ levels in these studies $[15,32]$ are not a matter of concern as chlorine disinfection is effective at $\mathrm{pH}$ less than 8 [37]. Turbidity affects the aesthetical values of drinking water as a result of visible cloudiness. To ensure effectiveness of disinfection, turbidity should be less than 1 NTU as recommended by the WHO [38]. Furthermore, water with high turbidity leads to health risk where it is ineffective against disinfectants as well as encourage bacterial growth during storage [39].

As for fluoride, studies by $[23,30,31]$ showed mean fluoride levels which were lower than the NDWQS. As for [23], some of the samples slightly exceeded the upper limit of $0.6 \mathrm{mg} /$ litre of the fluoride levels in the NDWQS. Fluoride is widely used in dental preparations to combat dental caries, particularly in areas on high sugar intake by which it can be in the form of tablets, mouth washers, toothpaste, varnishes or gels for local applications. It is also added in drinking water in order to provide protection against dental cavities [40]. The WHO recommends fluoride levels in drinking water to be between 0.9 to $1.2 \mathrm{mg} / \mathrm{L}$. In Malaysia, the Oral Health Division of the Ministry of Health has initiated the programme to add fluoride in drinking water since 1972. Initially, the recommended fluoride levels in Malaysian drinking water were 0.7 to $1.0 \mathrm{mg} / \mathrm{L}$. Considering the availability of alternative sources of fluoride and the higher average volume of water intake in a tropical climate, the levels have been reviewed to between 0.5 to $0.7 \mathrm{mg} / \mathrm{L}$ [41]. The NDWQS was revised recently and the fluoride levels permitted in drinking water are between 0.4 to $0.6 \mathrm{mg} / \mathrm{L}$.

In this review, studies reporting on chemical elements were very scarce. Only $14 \%$ out of the total number of studies included reported on chemical elements. For chemical parameters, pooled estimates were not able to be calculated as data was insufficient for each chemical parameters. Although some of the VOC compounds were reported by more than one studies, however results from [33] cannot be utilized for the computation of summary mean estimates because the study did not mention the number of water samples collected.

In general, measurements of VOC, THM and BPA reported by the researchers were within the stipulated guidelines of the NDWQS. VOC and THM levels were considered low since the levels were only about up to $20 \%$ of the recommended guideline values.

As for BPA, the parameter is not listed in the NDWQS hence no comparison against the guidelines can be made. Bisphenol A is a monomer used extensively in the production of polycarbonate, epoxy resins and as a non-polymer additive in plastics such as polyvinyl chloride (PVC) and water pipes [42]. Internal exposure to levels of unconjugated BPA may occur and poses risk to humans although BPA is rapidly biotransformed and excreted in urine [43]. While [44] found BPA at 0.1 and $1 \mathrm{nM}$ more effective at inhibiting the release of a key adipokine that protects humans from metabolic syndrome, higher exposure to BPA may also be associated with chronic health conditions such as diabetes and cardiovascular disease in humans [45]. Study by [29] reported that BPA was detected in all the tap water samples at levels ranging from 3.5 to $59.8 \mathrm{ng} / \mathrm{L}$ (mean: $14.1 \pm 14.36 \mathrm{ng} / \mathrm{L}$ ). BPA levels detected in tap water from European studies in comparison 
with [29] were lower ranging from not detected to $2 \mathrm{ng} / \mathrm{L}[46,47]$ whereas [48-50] reported higher BPA levels in water samples from Brazil and China at 160ng/L and 99ng/L. Inclusion of this endocrine disrupting chemicals (EDC) as one of the parameters in the guidelines is deemed essential. Many EDC-related diseases are currently on the rise and EDC are found in many products such as plastic bottles, detergents, flame retardant, food, toys and even cosmetics.

In terms of microorganisms, the number of studies conducted were very limited. However, based on those studies, it was found that the unfiltered water and underground water were highly contaminated. One of the study has revealed that all the unfiltered drinking water that were sampled were positive for coliforms, faecal coliforms and E. coil which concluded that the drinking water is not safe to be consumed and may be harmful to human health. The total viable count for the samples were high and did not comply with the safety regulation from European Council (EC) for drinking water. It was compared with EC as Food Act and most countries do not state the limit of total count for drinking water. Another study that was conducted on groundwater which is used as drinking water, similar results were found. $49 \%$ of the samples were positive for Total coliform, $14 \%$ positive for E. coli and $3 \%$ positive for Salmonella even though according to the National standard for Drinking water, Total Coliform and E. coli must be absent in drinking water. As for the presence of parasite in the water, only one study was conducted, and it was found that drinking or household water is free of parasites. However, it was shown that the untreated environmental water was contaminated with waterborne parasites.

The generally low number of available studies in this review which fulfil the inclusion criteria means that interpretation of results must be exercised with caution. Nevertheless, extensive effort in searching databases safeguards against missing relevant studies. The limitation of this review is that the search strategy was conducted only until May 2018 and does not consider any publications further than that timeline. Hence, relevant studies pertaining to drinking water quality in Malaysia might have been missing out and not included in this review.

\section{Conclusion}

Population growth and rapid industrialization advancement are some of the factors that increase pollutants in drinking water. In general, while some studies portray low level of contaminants and do not exceed the national drinking water guidelines, some studies slightly exceeded the stipulated guidelines. Although the overall water quality in Malaysia is fairly acceptable, we have to bear in mind that water pollution is rampant nowadays and measures have to be undertaken to safeguard the quality of drinking water through water treatment technology by water providers.

\section{Author Contributions}

All authors had complete access to the data in the study. NA contributed to the study concept and design. ZF and WNF acquired the data for the study. NA and WNF developed the search strategy. WR and NMS developed the pooled mean analysis. NIA and YV assisted with the analysis and interpretation of data. All authors were involved in the development of the manuscript. All authors critically revised the manuscript and approved the final version.

\section{Acknowledgement}

The authors would like to thank the Director General of Health, Malaysia for the permission to publish the study. The study was supported by National Institutes of Health (NIH). The NIH had no role in the design and conduct of the study; in the collection, analyses, or interpretation of data; in the writing of the manuscript, or in the decision to publish the results.

\section{References}

1. WHO/UNICEF (2000) Global Water Supply and Sanitation Assessment Report.

2. WHO/UNICEF (2015) Progress on Sanitation and Drinking Water: Update and MDG Assessment (2015).

3. Department of Environment Malaysia Environmental Quality Report (2017). Malaysia.

4. Azrina A, Khoo HE, Idris MA, Amn I, Razman MR (2011) Major inorganic elements in tap water samples in Peninsular Malaysia. Malays J Nutr 17(2): 271-276.

5. Aini MS, Fakhrul-Razi A, Mumtazah O, Chen JM (2007) Malaysian households' drinking water practices: a case study. Int J Sustain Dev World Ecol 14: 503-510.

6. (2013) Puncak Niaga Sdn. Bhd. (PNSB). Water treatment.

7. Ong C, Ibrahim S, Gupta BS (2007) A Survey of Tap Water Quality in Kuala Lumpur. Urban Water Journal 4(1): 29-41.

8. Ab Razak NH, Praveena SM, Aris AZ, Hashim Z (2016) Quality of Kelantan drinking water and knowledge, attitude and practice among the population of Pasir Mas, Malaysia. Public Health 131: 103-111.

9. Abdullah MP, Soh SC (2011) Chlorinated and Nonchlorinated-Volatile Organic Compounds (VOCS) in Drinking Water of Peninsular Malaysia. Sains Malaysiana 40(11): 1255-1261.

10. Abdullah MP, Yew CH, Mohammad Salleh R (2003) Formation, modeling and validation of trihalomethanes (THM) in Malaysian drinking water: a case study in the districts of Tampin, Negeri Sembilan and Sabak Bernam, Selangor, Malaysia. Water Res 37(19): 4637-4644.

11. Hassimi AH, Siti Rozaimah SA, Siti Kartom K, Noorhisham TK (2011) Problems of Ammonia and Manganese in Malaysian Drinking Water Treatments. World Applied Sciences Journal, pp. 1890-1896.

12. Nisar A, Mohamad SJ, Mohammed SA (2015) Study of radon concentration and toxic elements in drinking and irrigated water and its implications in Sungai Petani, Kedah, Malaysia. Journal of Radiation Research and Applied Science 8(3): 294-299.

13. Anita DK, Surfina AS, Jack ZT, Linn WM, Shazneen FB (2015) Assessment of Drinking Water Quality in a Community in Malaysia. Asian Journal of Water, Environment and Pollution 12(4): 11-15. 
14. Azlan A, Khoo HE, Mohd AI, Amin I, Muhammad RR (2012) Evaluation of minerals content of drinking water in Malaysia. Scientific World Journal 2012(403574): 10.

15. Chan CL, Zalifah MK, Norrakiah A (2007) Microbiological and Physiochemical Quality of Drinking Water. The Malaysian Journal of Analytical Sciences 11(2): 414-420.

16. Mohd AD, Mohd SS, Abdul AM, Aizat IS (2011) Risk Assessment of Aluminium in Drinking Water between Two Residential Areas. Water 3(3): 882-893.

17. Idrus AS, Fauziah MN, Hani MH, Wan Rohaila WA, Wan Manzor H (2014) Status of Groundwater Contamination in Rural Area, Kelantan. Journal of Environmental Science, Toxicology and Food Technology $8(1): 72-80$.

18. Noorain MI, Ahmad ZA, Wan YL, Wan NAWS, Praveena SM (2014) Evaluation of Heavy Metal Contamination in Groundwater Samples from Kapas Island, Terengganu Malaysia. Arab J Geoscience 7(3):10871100.

19. Khoo HE, Azrina A, Mohd AI, Amin I, Muhammad RR (2011) Evaluation of Selected Metal Elements in Commercial Drinking Water and Tap Water in Peninsular Malaysia Jurnal Sains Kesihatan Malaysia 9(1): 5-11.

20. Kumar T, Onichandran S, Yvonne ALL, Sawangjaroen N, Ithoi I, et al. (2014) Comparative study on waterborne parasites between Malaysia and Thailand: A new insight. Am J Trop Med Hyg 90(4): 682-689.

21. Lim CS, Shaharuddin MS, Sam WY (2013) Risk Assessment of Exposure to Lead in Tap Water among Residents of Seri Kembangan, Selangor State, Malaysia. Global Journal of Health Science 5(2): 1-12.

22. Mohd Hasni J, Aminuddin M, Ariza Z, Azwani A, Engku Nurul Syuhadah EA, et al. (2017) Comparison of Heavy Metal Levels in Natural Spring and Bottled Drinking Water in Klang Valley, Malaysia. Malaysian Journal of Public Health Medicine 17(1): 46-50.

23. Shaharuddin MS, Yusoff MK, Yaziz MI (2009) Fluoride Concentration in Malaysian Drinking Water American-Eurasian J. Agric \& Environ Sci 6(4): 417-420.

24. Nalatambi S (2009) Determination of Metals in Tap Water using Atomic Absorption Spectrometry: A case study in Bandar Sunway residential area. Sunway Academic Journal 6: 33-46.

25. Praveena SN, Nurul FKH, Claire dB (2018) Public health risk assessment from drinking water from vending machines in Seri Kembangan (Malaysia). Food Control 91: 40-46.

26. Qaiyum MS, Shaharuddin MS, Syazwan AI, Muhaimin A (2011) Health risk assessment after exposure to aluminium in drinking water between two different villages. Journal of Water Resources and Protection 3: 268-274.

27. Rahmanian N, Siti Hajar A, Jomayoonfard M, Ali NJ, Rehan M, et al. (2015) Analysis of Physiochemical Parameters to Evaluate the Drinking Water Quality in the State of Perak, Malaysia. Journal of Chemistry 2015(716125): 1-10.

28. Richard RL, Ithoi I, Abd Majid MA, Wan Sulaiman WY, Tan TC, et al. (2016) Monitoring of waterborne parasites in two drinking water treatment plants: A study in Sarawak, Malaysia. Int J Environ Res Public Health 13(7): 641-662.

29. Santhi VA, Sakai N, Ahmad ED, Mustafa AM (2012) Occurrence of Bisphenol A in surface water, drinking water and plasma from Malaysia with exposure assessment from consumption of drinking water. Sci Total Environ 427-428: 332-338.

30. Shaharuddin MS, Nor Kidahus MM, Sumarlan S, Mohd Kamil Y, et al. (2010) Dental Fluorosis (DF) and Its Relationship with fluoride Levels in Drinking Water in Three States in Malaysia. Research Journal of Medical Sciences 4(1): 20-24.

31. Shaharuddin MS, Mohd Kamil Y, Mohamed Ismail Y, Mohammad Firuz R, Mohd Yunus A, et al. (2010) Fluoride levels in drinking water from nine study sites in Peninsular Malaysia and Sabah Health and the Environment Journal 1(1): 51-54.

32. Siti Farizwana MR, Mazrura S, Zurahanim Fasha A, Ahmad Rohi G (2010) Determination of aluminium and physicochemical parameters in the palm oil estates water supply at Johor, Malaysia. J Environ Public Health.

33. Soh SC, Md Pauzi A (2007) Determination of volatile organic compounds pollution sources in Malaysian drinking water using multivariate analysis. Environ Monit Assess 124(1-3): 39-50.

34. Tan EY, Mohammed A, Soon JM (2016) Identification of Escherichia Coli strains from water vending machines of Kelantan, Malaysia using $16 \mathrm{~S}$ rRNA gene sequence analysis. Expo Health.

35. Yusof AM, Mahat MN, Omar N, Wood AKH (2001) Water quality studies in an aquatic environment of disused tin-mining pools and in drinking water Ecologial Engineering 16(3): 405-414.

36. Shao Q Wang YG (2009) Statistical power calculation and sample size determination for environmental studies with data below detection limits. Water Resources Research 45(9): 1-8.

37. (2007) World Health Organization (WHO). Guidelines for Drinkingwater Quality.

38. (2011) World Health Organization (WHO). Guidelines for drinkingwater quality ( $4^{\text {th }}$ edn).

39. Hoko Z (2005) An assessment of the water quality of drinking water in rural districts in Zimbabwe. The case of Gokwe South, Nkayi, Lupane, and Mwenezi districts. Physics and Chemistry of the Earth, Parts A/B/C 30(11-16): 859-866.

40. (2008) World Health Organization (WHO) Guidelines for drinkingwater quality: second addendum. Vol 1, Recommendations. $-\left(3^{\text {rd }}\right.$ edn $)$.

41. (2002) Ministry of Health Malaysia. Annual Report.

42. Huang G, Zhuo A, Wang L, Wang X (2011) Preparation and flammability properties of intumescent flame retardant-functionalized layered double hydroxides/polymethyl methacrylate nanocomposites. Material Chemistry and Physiscs 130(1-2): 714-720.

43. Welshons WV, Nagel SC, vom Saal FS (2006) Large effects from small exposures. III. Endocrine mechanisms mediating effects of bisphenol A at levels of human exposure. Endocrinology 147 (6 Suppl): S56-S69.

44. Vandenberg LN, Chahoud I, Heindel JJ, Padmanabhan V, Paumgartten FJ, et al. (2010) Urinary, circulating, and tissue biomonitoring studies indicate widespread exposure to bisphenol A. Env Heal Perspect 118: 1055-1070.

45. Hugo ER, Brandebourg TD, Woo JG, Loftus J, Alexander JW, et al. (2008) Bisphenol A at environmentally relevant doses inhibits adiponectin release from human adipose tissue explants and adipocytes. Environ Health Perspect 116(12): 1642-1647.

46. Lang IA, Galloway TS, Scarlett A, Henley WE, Depledge M, et al. (2008) Association of urinary bisphenol A concentration with medical disorders and laboratory abnormalities in adults. JAMA 300(11): 1303-1310.

47. Kuch HM, Ballschmiter K (2001) Determination of endocrinedisrupting phenolic compounds and estrogens in surface and drinking water by HRGC-(NCI)-MS in the picogram per liter range. Environ Sci Technol 35(15): 3201-3206. 
48. Loos R, Hanke G, Umlauf G, Eisenreich SJ (2007) LC-MS-MS analysis and occurrence of octyl- and nonylphenol, their ethoxylates and their carboxylates in Belgian and Italian textile industry, waste water treatment plant effluents and surface waters. Chemosphere 66(4): 690-699.

49. Sodré FF, Locatelli MAF, Jardim WF (2010) Occurrence of Emerging
Contaminants in Brazilian Drinking Waters: A Sewage-To-Tap Issue. Water Air Soil Pollut 206: 57-67.

50. Li X, Ying GG, Su HC, Yang XB, Wang L (2010) Simultaneous determination and assessment of 4-nonylphenol, bisphenol $\mathrm{A}$ and triclosan in tap water, bottled water and baby bottles Environment International 36(6): 557-562.

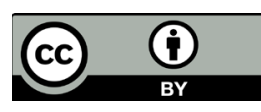

his work is licensed under Creative Commons Attribution 4.0 License

DOI:10.19080/IJESNR.2020.24.556132 